W ährend die im Jahr 2012 erstellte Treat-to-Target-Empfehlung $\mathrm{zu}$ Spondylarthritiden (SpA) fast ausschließlich eminenzbasiert war, kann die in Madrid vorgestellte Aktualisierung als weitgehend evidenzbasiert gelten. Dies berichtete Prof. Désirée van der Heijde, Leiden, NL. Unter den übergeordneten Prinzipien wird unter anderem betont, dass die Strategie, auf Ziel zu behandeln, also die Krankheitsaktivität in bestimmten Zeitabständen zu überprüfen und die Therapie bei Bedarf anzupassen, nachweislich zu einem besseren Ergebnis führt. Ziel sollte eine Remission (keine klinischen oder Laborhinweise auf eine Krankheitsaktivität) oder zumindest eine niedrige Aktivität sein. Für die axiale $\mathrm{SpA}(\mathrm{axSpA})$ sollte der ASDAS (Ankylosing Spondylitis Disease Activity Score) als Maßstab gewählt werden, da er mit der Bildung von Syndesmophyten und damit den körperlichen Einschränkungen und einer Verringerung der Lebensqualität korreliert.
Als zusätzliches Beurteilungskriterium kann die Bildgebung genutzt werden, wobei die Low-Dose-Computertomografie wohl zukünftig die Methode der Wahl bei der axSpA sein wird. In die Beurteilung der Krankheitsaktivität sollten zudem Patienten-relevante Faktoren, extraartikuläre Manifestationen wie die anteriore Uveitis und Komorbiditäten wie Fatigue einfließen. Schließlich sollten die Therapieziele und -strategien gemeinsam mit dem Patienten besprochen und getroffen werden.

\title{
Neue Therapieoptionen bei RA
}

$\mathrm{D}$ er gerade in der EU zugelassene Interleukin 6(IL6)-Rezeptor-Inhibitor Sarilumab und der direkte IL-6-Inhibitor Sirukumab, dessen Zulassung im Frühjahr 2018 erwartet wird, ergänzen die Palette der IL6-Inhibitoren. In Madrid wurden die Ergebnisse zu „patient reported outcomes (PRO) unter Sarilumab im Vergleich zu Adalimumab, jeweils als Monotherapie nach MTX-Versagen oder bei MTX-Intoleranz, vorgestellt. Der IL6-Inhibtor führte mehrheitlich $\mathrm{zu}$ einer stärkeren und klinisch relevanten Verbesserung im Vergleich zum TNF- $\alpha$-Inhibitor, so Dr. Vibeke Strand, Palo Alto, USA. Dies galt für die vom Patienten berichtete Krankheitsak- tivität ebenso wie für Schmerzen, körperliche Funktion, Morgensteifigkeit, Arbeitsfähigkeit und die gesundheitsbezogene Lebensqualität.

Die JAK-Inhibitoren Baricitinib und Tofacitinib haben es dagegen schon in den klinischen Alltag der Rheumatherapie geschafft. Sie können bei RA-Patienten mit mittelschwerer bis schwerer Krankheitsaktivität aufgrund eines nicht ausreichenden Ansprechens auf mindestens ein csDMARD in der Kombination mit Methotrexat (MTX) verordnet werden, bei MTX-Unverträglichkeit in der Monotherapie. In der aktualisierten EULAR-Leitlinie haben sie schon einen festen Platz.

\section{Intradiskale}

\section{Steroidinjektionen \\ wirken nur kurz}

D atienten mit chronischen Schmerzen im Bereich der Lendenwirbelsäule profitieren nur kurzfristig von intradiskalen Steroidinjektionen, wie eine französische Studie ergab. Darin hatten $\mathrm{Pa}$ tienten mit aktiver Diskopathie in der MRT entweder einmalig eine Spritze mit $25 \mathrm{mg}$ Prednisolonacetat oder mit Placebo erhalten. Einen Monat nach der Intervention war zwar der Schmerzwert auf der visuellen Analogskala bei 84,6\% versus 54,0\% unter 40. Nach einem Jahr fand sich aber kein Unterschied mehr [Abstract LB0001]. 